\title{
TORSION UNITS IN INTEGRAL GROUP RINGS
}

\author{
ANGELA VALENTI \\ (Communicated by Ronald M. Solomon)
}

\begin{abstract}
Let $G=\langle a\rangle \rtimes X$ where $\langle a\rangle$ is a cyclic group of order $n, X$ is an abelian group of order $m$, and $(n, m)=1$. We prove that if $\mathbb{Z} G$ is the integral group ring of $G$ and $H$ is a finite group of units of augmentation one of $\mathbb{Z} G$, then there exists a rational unit $\gamma$ such that $H^{\gamma} \subseteq G$.
\end{abstract}

Let $G$ be a finite group, $\mathbb{Z} G$ the integral group ring of $G$, and $U_{1} \mathbb{Z} G$ the group of units of augmentation one in $\mathbb{Z} G$. It has been conjectured by Zassenhaus that if $H$ is a finite subgroup of $U_{1} \mathbb{Z} G$, then $H$ is conjugate to a subgroup of $G$ by a rational unit, i.e., there exists $\gamma \in U \mathbb{Q} G$ such that $H^{\gamma} \subseteq G$.

This conjecture has been confirmed by Weiss in [8] for $p$-groups. In this note we shall prove this conjecture for a certain class of metabelian groups. More precisely, we will establish the following result:

Theorem. Let $G$ be a split extension $\langle a\rangle \rtimes X$, where $\langle a\rangle$ is a cyclic group of order $n, X$ is an abelian group of order $m$, and $(n, m)=1$. If $H \subseteq U_{1} \mathbb{Z} G$ is a finite group, then there exists $\gamma \in U \mathbb{Q} G$ such that $H^{\gamma} \subseteq G$.

Before proceeding to the proof of the theorem we record some useful facts that will be needed.

If $N$ is a normal subgroup of $G$, let us denote by $\Delta(G, N)$ the kernel of the natural map $\mathbb{Z} G \rightarrow \mathbb{Z}(G / N)$. Also we briefly write $u \sim g$ to indicate that $u$ is conjugate in $\mathbb{Q} G$ to $g$.

Lemma 1. Let $G=A \rtimes X$, where $A$ is an abelian normal p-group and $X$ is any group with $(|A|,|X|)=1$. Let $u \in U_{1} \mathbb{Z} G$ be a unit of the form $u=v w$, where $v \in U(1+\Delta(G, A)), w \in U_{1} \mathbb{Z} X$. If $u$ has finite order not divisible by $p$, then $u \sim w$.

Proof. See [3, Lemma 2].

Lemma 2. Let $G=\langle a\rangle \rtimes X$, where $o(a)=n,|X|=m$, and $(n, m)=1$. If an element $a^{i} x$ of $G$, where $x \in X$, is of order divisible by all primes dividing $n$, then $x$ is central in $G$.

Proof. It is a consequence of [4, Lemma 2.3].

Lemma 3. Let $G$ be a split extension $\langle a\rangle \rtimes X$, where $\langle a\rangle$ is a cyclic group of order $n, X$ is an abelian group of order $m$, and $(n, m)=1$. If $G_{0}$ is a

Received by the editors June 18, 1991

1991 Mathematics Subject Classification. Primary 20C05; Secondary 16S34. 
subgroup of $G$, then $G_{0}=\langle b\rangle \rtimes X_{0}$, where $b \in\langle a\rangle$ and $X_{0}$ is isomorphic to a subgroup of $X$.

Proof. If $\varphi: G_{0} \rightarrow X$ is such that $a^{i} x \rightarrow x$, then $\varphi$ is a homomorphism and $\operatorname{Ker} \varphi=\langle b\rangle$ for some $b \in\langle a\rangle$. Hence $G_{0} / \operatorname{Ker} \varphi \cong \varphi\left(G_{0}\right)$ and $G_{0}=\langle b\rangle \rtimes X_{0}$, with $X_{0}$ isomorphic to a subgroup of $X$.

The proof of the theorem will be based on the following reduction:

Lemma 4. Let $G$ be a finite group, $G_{0}$ a subgroup of $G$, and $H$ a finite subgroup of $U \mathbb{Z} G$. Suppose that there exists an isomorphism $\varphi: H \rightarrow G_{0}$ such that, for all $h \in H$ and for all complex irreducible characters $\chi$ of $G, \chi(h)=\chi(\varphi(h))$. Then $G_{0} \sim H$.

Proof. This has been proved when $H$ is a cyclic subgroup in [4, 5]. The same argument gives a proof in general. See also [7, Lemma 4.6].

We can now prove the main result of this note.

Proof of the theorem. Let $G=\langle a\rangle \rtimes X$, where $o(a)=n,|X|=m$, and $(n, m)=1$; also let $H$ be a finite subgroup of $U_{1} \mathbb{Z} G$. In order to prove the theorem we will construct an isomorphism $\varphi$ of $H$ onto a subgroup of $G$ satisfying the criterion of Lemma 4 ; that is, $\varphi$ will be such that, for all irreducible characters $\chi$ of $G, \chi(h)=\chi(\varphi(h))$ for all $h \in H$.

By Whitcomb's argument given on [6, p. 103] it follows that, for all $h \in H$, there exists an element $g_{h}$ of $G$ such that $h \equiv g_{h}(\bmod \Delta G \Delta\langle a\rangle)$. Since in the metabelian group case being considered it has been proved by Cliff, Sehgal, and Weiss in [1] that $U(1+\Delta(G,\langle a\rangle))$ is torsion-free, it follows that the torsion subgroup $H$ of $U_{1} \mathbb{Z} G$ is isomorphic to a subgroup $G_{0}$ of $G$. Let $\alpha: H \rightarrow G_{0}$ be the above isomorphism defined by $\alpha(h)=g_{h}$. By Lemma 3, $G_{0}=\langle b\rangle \rtimes X_{0}$ where $b \in\langle a\rangle$ and $X_{0}$ is a sroup isomorphic to a subgroup of $X$. Taking preimages, we can write $H=\langle u\rangle \rtimes K$, where $\alpha(u)=b$ and $\alpha(K)=X_{0}$.

Since $o(u)$ divides $o(a)$, by [2, Theorem 1.1], we have that $u \sim g$ for some $g \in\langle a\rangle$. Also, since $\alpha$ is an isomorphism and $u \sim g$, we have $o(g)=o(u)=$ $o(b)$. Hence $g=b^{i}$ for some $i$ and $u \sim b^{i}$.

Now let $k \in K$. We have $\alpha(k)=a^{j} x$ for some $j$ and some $x \in X$. Note that if $k_{1} \neq k_{2}$ and $\alpha\left(k_{1}\right)=a^{j_{1}} x, \alpha\left(k_{2}\right)=a^{j_{2}} x$, then $x_{1} \neq x_{2}$ (since otherwise $\alpha\left(k_{1} k_{2}^{-1}\right) \in\langle a\rangle$, contradicting the relative primeness of $|K|$ and $\left.o(a)\right)$.

We shall prove by induction on the number of different primes dividing $o(a)$ that $k \sim x$. Since $k \equiv a^{j} x(\bmod \Delta G \Delta\langle a\rangle)$, it follows that $k=(1+\delta) x$ for some $\delta \in \Delta G \Delta\langle a\rangle$. Hence, if $\langle a\rangle$ is a $p$-group, since $(o(k), o(a))=1$, we obtain, by Lemma 1 , that $k \sim x$, and we are done in this case.

Now let in general $o(a)=p_{1}^{n_{1}} \cdots p_{t}^{n_{t}}$, and write $\langle a\rangle=\left\langle a_{1}\right\rangle \times\left\langle a_{2}\right\rangle$, where $o\left(a_{1}\right)=p_{1}^{n_{1}}$ and $o\left(a_{2}\right)=p_{2}^{n_{2}} \cdots p_{t}^{n_{t}}$. Then $G=\left\langle a_{1}\right\rangle \rtimes G_{2}$, where $G_{2}=$ $\left\langle\left\langle a_{2}\right\rangle, X\right\rangle$, and we have that $U_{1} \mathbb{Z} G=U\left(1+\Delta\left(G,\left\langle a_{1}\right\rangle\right)\right) \rtimes U_{1} \mathbb{Z} G_{2}$. If we write $k=\left(1+\delta_{1}\right) \gamma$, where $\left(1+\delta_{1}\right) \in U\left(1+\Delta\left(G,\left\langle a_{1}\right\rangle\right)\right)$ and $\gamma \in U_{1} \mathbb{Z} G_{2}$, then, by Lemma 1, it follows that $k \sim \gamma$. On the other hand, we can write $\gamma=\left(1+\delta_{2}\right) y$, where $\left(1+\delta_{2}\right) \in U\left(1+\Delta\left(G_{2},\left\langle a_{2}\right\rangle\right)\right)$ and $y \in U_{1} \mathbb{Z} X=X$. But then by the inductive hypothesis $\gamma \sim y$, and this forces $k \sim y$. We now claim $x=y$. In fact, since $\left(1+\delta_{1}\right) \equiv a_{1}^{t}\left(\bmod \Delta G \Delta\left\langle a_{1}\right\rangle\right)$ for some $t$ and $\gamma=\left(1+\delta_{2}\right) y \equiv$ $a_{2}^{s} y\left(\bmod \Delta G_{2} \Delta\left\langle a_{2}\right\rangle\right)$ for some $s$, we have $k=\left(1+\delta_{1}\right)\left(1+\delta_{2}\right) y \equiv a_{1}^{t} a_{2}^{s} y$ $(\bmod \Delta G \Delta\langle a\rangle)$. In particular, $a_{1}^{t} a_{2}^{s} y \equiv a^{j} x(\bmod \Delta G \Delta\langle a\rangle)$. By [1] it follows that $a_{1}^{t} a_{2}^{s} y=a^{j} x$, and so $x=y$, as claimed. 
We now define a map $\varphi: H \rightarrow G$ by setting $\varphi(u)=b^{i}$ and $\varphi(k)=x$, where $k \in K$ and $\alpha(k)=a^{j} x$ for some $j$. It is easy to see that $\varphi$ is a homomorphism, and our earlier argument tells us that $\varphi$ is one-to-one. The conjugacy results obtained above allow us to conclude that $\chi(u)=\chi(\varphi(u))$ and $\chi(k)=\chi(\varphi(k))$ for all $k \in K$ and all irreducible characters $\chi$ of $G$.

We want to prove that, for every $h \in H$ and for every irreducible character $\chi$ of $G, \chi(h)=\chi(\varphi(h))$. With this, the proof of the theorem will be completed according to Lemma 4 . To this effect we use induction on the number of different primes dividing $o(a)$ but not dividing $o(h)$.

Let $h=u^{t} k \in H$ for some $k \in K$. If $o(h)$ is divisible by all primes dividing $o(a)$, then, since $o\left(b^{i t} x\right)=o(\varphi(h))=o(h)$, by Lemma 2, $x$ is central in $G$. Hence, since $k \sim x, k=x$. Thus $h=u^{t} k$ and $\varphi(h)=b^{i t} x$ are conjugate, and this says that $\chi(h)=\chi(\varphi(h))$ for all irreducible characters $\chi$ of $G$. Therefore, we may assume that at least one of the primes dividing $o(a)$, say $p$, does not divide $o(h)$.

As above write $\langle a\rangle=\left\langle a_{1}\right\rangle \times\left\langle a_{2}\right\rangle$, where $\left\langle a_{1}\right\rangle$ is a $p$-group and $\left\langle a_{2}\right\rangle$ is a $p^{\prime}$-group. Then $G=\left\langle a_{1}\right\rangle \rtimes G_{2}$, where $G_{2}=\left\langle\left\langle a_{2}\right\rangle, X\right\rangle$, and we have $U_{1} \mathbb{Z} G=$ $U\left(1+\Delta\left(G,\left\langle a_{1}\right\rangle\right)\right) \rtimes U_{1} \mathbb{Z} G_{2}$. Moreover, $h=(1+\delta) \gamma$, where $(1+\delta) \epsilon$ $U\left(1+\Delta\left(G,\left\langle a_{1}\right\rangle\right)\right)$ and $\gamma \in U_{1} \mathbb{Z} G_{2}$. By Lemma 1, it follows that $h \sim \gamma$, so $\chi(h)=\chi(\gamma)$ for all irreducible characters $\chi$ of $G$.

Let us denote by $\bar{\varphi}$ the homomorphism induced by $\varphi$ when we factorize by $\left\langle a_{1}\right\rangle$, i.e.,

$$
\bar{\varphi}: \bar{H}=\langle\bar{u}\rangle \rtimes \bar{K} \rightarrow \bar{G}=G_{2} .
$$

Our map behaves well with respect to factoring by $\left\langle a_{1}\right\rangle$; namely $\bar{\varphi}(\bar{u})=\bar{b}^{i}$ and $\bar{\varphi}(\bar{k})=\bar{x}$.

Since $h=\left(1+\delta_{1}\right) \gamma$, where $\left(1+\delta_{1}\right) \in U\left(1+\Delta\left(G,\left\langle a_{1}\right\rangle\right)\right)$ and $\gamma \in U_{1} \mathbb{Z} G_{2}$, by factoring by $\left\langle a_{1}\right\rangle$ we get $\bar{h}=\gamma$, so $\bar{\varphi}(\bar{h})=\bar{\varphi}(\bar{\gamma})=\bar{b}^{i t} \bar{x}$.

By the inductive hypothesis, $\psi(\bar{\varphi}(\bar{h}))=\psi(\bar{h})=\psi(\gamma)=\psi\left(\bar{b}^{i t} \bar{x}\right)$ for every irreducible character $\psi$ of $G_{2}=A_{2} \rtimes X$. But if $\chi$ is an irreducible character of $G$ and $\chi_{G_{2}}$ is the induced character on $G_{2}$, then $\chi_{G_{2}}$ is a linear combination of irreducible $G_{2}$-characters, and by the previous argument it follows that $\chi(\gamma)=$ $\chi_{G_{2}}(\gamma)=\chi_{G_{2}}\left(\bar{b}^{i t} \bar{x}\right)=\chi\left(\bar{b}^{i t} \bar{x}\right)$. Finally, by writing $b^{i t} x=a_{1}^{l} \bar{b}^{i t} \bar{x}$, for some $l$, since $p$ does not divide $o(h)=o\left(b^{i t} x\right)$, it follows, by Lemma 1, that $b^{i t} x \sim$ $\bar{b}^{i t} \bar{x}$. Thus $\chi(h)=\chi(\gamma)=\chi\left(\bar{b}^{i t} \bar{x}\right)=\chi\left(b^{i t} x\right)$ for every irreducible character $\chi$ of $G$. This completes the proof of the theorem.

\section{REFERENCES}

1. G. H. Cliff, S. K. Sehgal, and A. R. Weiss, Units of integral group rings of metabelian groups, J. Algebra 73 (1981), 167-185.

2. I. S. Luthar and P. Trama, Zassenhaus conjecture for certain integral group rings, J. Indian Math. Soc. 55 (1990), 199-212.

3. C. P. Milies and S. K. Sehgal, Torsion units in integral group rings of metacyclic groups, J. Number Theory 19 (1984), 103-114.

4. C. P. Milies, J. Ritter, and S. K. Sehgal, On a conjecture of Zassenhaus on torsion units in integral group rings. II, Proc. Amer. Math. Soc. 97 (1986), 201-206.

5. J. Ritter and S. K. Sehgal, On a conjecture of Zassenhaus on torsion units in integral group rings, Math. Ann. 264 (1983), 257-270.

6. S. K. Sehgal, Topics in group rings, Dekker, New York, 1978. 
7. __ Lectures on group rings, Univ. of Palermo, 1991.

8. A. Weiss, Rigidity of p-adic p-torsion, Ann. of Math. (2) 127 (1988), 317-332.

Dipartimento di Matematica ed Applicazioni, Università di Palermo, Via Archirafi 34, 90123 Palermo, Italy 